\title{
CIRURGIA REDUTORA DE VOLUME PULMONAR: CRITÉRIOS DE SELEÇÃO DE PACIENTES NO HOSPITAL UNIVERSITÁRIO ANTÔNIO PEDRO, UNIVERSIDADE FEDERAL FLUMINENSE, NITERÓI, RIO DE JANEIRO, RJ
}

\author{
Rodolfo fred Behrsin*, Cyro Teixeira da Silva Junior, José Manoel Gabetto, \\ Oriane de Almeida Santana lima \\ Trabalho realizado no Ambulatório Multidisciplinar de DPOC e Tabagismo, vinculado aos Cursos de \\ Pós-graduação em Pneumologia da Universidade Federal Fluminense, Niterói, Rio de Janeiro, com \\ participação do Setor de Fisiopatologia Cardiopulmonar e Serviço de Imagem do Hospital Universitário \\ Antônio Pedro, Niterói, Rio de Janeiro, RJ.
}

RESUMO - 0 tratamento clínico da doença pulmonar obstrutiva crônica (DPOC) está bem padronizado por consensos nacionais e internacionais. Junto com transplante pulmonar, a cirurgia redutora de volume pulmonar (CRVP) é alternativa de tratamento cirúrgico para o enfisema.

OBjetivos. Avaliar critérios de inclusão e exclusão para realização de CRVP em pacientes com DPOC.

Métodos. Trinta e um pacientes encaminhados e acompanhados no Ambulatório Multidisciplicar de Pesquisa em DPOC e Tabagismo dos Cursos de Pós-Graduação em Pneumologia da Universidade Federal Fluminense, submetidos a um protocolo de avaliação para seleção de candidatos à CRVP que incluiu anamnese e exame físico padronizados, espirometria com curva fluxo volume e teste com broncodilatador, gasometria arterial com paciente respirando ar ambiente, teste de caminhada por seis minutos (TC6M), radiografia convencional de tórax em póstero anterior e perfil esquerdo, tomografia computadorizada de tórax de alta resolução (TCAR) e cintilografia pulmonar de ventilação e perfusão. Análise estatística: descritiva.

RESULTADOs. Contra-indicações para CRVP: bronquite crônica
( $29 \%$ ou nove pacientes), VEF I $\geq 80,0 \%$ do previsto (I3\% ou quatro pacientes), recusa em realizar a cirurgia (10\% ou três pacientes), áreas de fibrose, depressão e bronquiectasias (dois pacientes cada), nódulo pulmonar solitário neoplásico, obesidade mórbida, enfisema bolhoso grave, hipertensão pulmonar, passado de ressecção pulmonar, tabagismo corrente, enfisema difuso com deficiencia de alfa-Iantitripsina e doença cardiaca grave (um paciente cada). Um dos pacientes selecionados concordou e foi submetido à CRVP. Sua indicação foi enfisema pulmonar heterogênio com predomínio nos lobos superiores, diagnosticado pela TCAR e cintilografia pulmonar.

Conclusões. Bronquite crônica representou a principal contra-indicação em $29 \%$ dos casos. Pacientes com indicação de CRVP, freqüentemente, apresentam mais de um fator mórbido a contra-indicar o seu procedimento. A maioria dos pacientes com critérios para CRVP, a recusa, com receio do procedimento ou após programas de reabilitação pulmonar. 0 critério mais significativo que indicou CRVP em um paciente operado foi a presença de enfisema heterogênio bilateral nos lobos superiores.

UNITERMOS: Cirurgia redutora de volume pulmonar. Enfisema pulmonar.

\section{INTRODUÇÃO}

A cirurgia redutora de volume pulmonar (CRVP) foi originalmente proposta por Otto Brantigan em 1959', porém, não conseguindo explicar o mecanismo de melhora dos pacientes e apresentando uma elevada taxa de mortalidade, não encontrou aceitação, sendo abandonada e considerada dado histórico por vários anos². Em 1995, Cooper, auxiliado por novas tecnologias (TCAR, cintilografia pulmonar de ventilação e perfusão, além de cuidados de terapia inten-

\footnotetext{
* Correspondência

Alameda Aleides, 76 24230-120 - Niterói - RJ
}

siva no pós operatório) inexistentes em 1959, realizou 20 CRVP com excelentes resultados, despertando atenção sobre este procedimento ${ }^{3}$. Tal interesse pode ser justificado pelos bons resultados apresentados, como redução da hipoxemia e da dependência de oxigênio, redução da retenção de gás carbônico, melhora da qualidade de vida e da capacidade de realizar exercícios por parte dos pacientes, além de baixa taxa de mortalidade. É considerada a única abordagem da doença pulmonar obstrutiva crônica (DPOC) capaz de melhorar significativamente os parâmetros da espirometria ${ }^{4}$.

O presente trabalho teve os seguintes objetivos: estudar os critérios de inclusão e de exclusão de pacientes candidatos a CRVP.

\section{Métodos}

Entre dezembro de 1997 e março de 1999 foram encaminhados 65 pacientes, oriundos de diferentes serviços. Desta amostra, 34 pacientes foram reencaminhados aos serviços de origem, após avaliação e início de tratamento, onde deram continuidade ao seu acompanhamento médico, não sendo portadores de doença pulmonar obstrutiva crônica.

Os 31 pacientes restantes compreenderam um grupo de 29 homens e duas mulheres com idade média de 60,77 anos (o mais idoso com 77 anos e o mais jovem com 39 anos). Todos apresentavam alguma relação com o tabagismo, sendo 30 ex-tabagistas e um tabagista corrente, mostrando uma média de 47,63 maços/ano. 
Os pacientes foram submetidos a protocolo de avaliação que incluiu: anamnese, exame físico, radiografias convencionais do tórax em incidências póstero-anterior e de perfil, tomografia computadorizada de tórax de alta resolução(TCAR) em inspiração, sendo realizados dez cortes desde o ápice ao diafragma com espessura de $2 \mathrm{~mm}$ cada e sem o emprego de contraste, espirometria com valorização da curva fluxo-volume e teste pós-broncodilatador, teste de caminhada por seis minutos (TC6M), gasometria arterial e cintilografia pulmonar de ventilação e perfusão.

As TCAR foram analisadas por um médico radiologista com o objetivo de se quantificar o enfisema pulmonar, utilizando-se método descrito por Bergin et al. ${ }^{5}$. Cada hemitórax de cada corte tomográfico foi analisado e recebeu um determinado valor baseado na área de secção transversa comprometida pelo enfisema pulmonar: 0 - sem presença de enfisema pulmonar visível; I - menos de $25 \%$ de comprometimento de secção transversa; 2 - comprometimento entre $25 \%$ a $50 \%$ da secção transversa; 3 - comprometimento entre $50 \%$ a 75\% da secção transversa; 4 - mais de 75\% de comprometimento da secção transversa. Após avaliação de todos os cortes tomográficos, os valores foram somados sendo 0 valor máximo possível encontrado igual a 80 .

$\mathrm{Na}$ espirometria foram considerados os valores de capacidade vital forçada (CVF), volume expiratório forçado no primeiro segundo (VEF I) e relação VEF /CVF, além da resposta a broncodilatador (fenoterol - 400 $\mathrm{mcg}$ ) e realizada gasometria arterial com paciente respirando ar ambiente.

Realizou-se teste de TC6M, o qual foi conduzido sempre pelo mesmo médico pneumologista, sendo efetuado pelo menos dois testes por paciente, com intervalo mínimo de 15 dias e máximo de 21 dias entre cada avaliação, e considerado o melhor resultado.

Os pacientes que apresentaram avaliação final favorável à realização de CRVPe manifestaram interesse por sua realização, após o oferecimento desta alternativa terapêutica, foram então submetidos à realização de cintilografia pulmonar de ventilação e perfusão com o objetivo de obter um mapeamento mais preciso das áreas a serem ressecadas.

Análise estatística: descrição da análise dos resultados, porque trata-se de estudo aberto, não comparativo.

\section{Resultados}

A análise dos 31 pacientes resultou nas seguintes contra-indicações para a CRVP: bronquite crônica ( $29 \%$ ou nove pacientes), VEFI maior ou igual a $80 \%$ do previsto (I $3 \%$ ou quatro pacientes), recusa em realizar a cirurgia ( $10 \%$ ou três pacientes), áreas de fibrose, depressão grave e bronquiectasias (dois pacientes cada). Outras contra-indicações, com um paciente cada, foram nódulo neoplásico pulmonar, enfisema bolhoso extenso, obesidade mórbida, hipertensão arterial pulmonar, toracotomia antiga para ressecção de nódulo pulmonar neoplásico, deficiência de alfa- I-antitripsina, tabagista corrente com recusa de abandonar o vício e insuficiência coronariana. Somente um paciente selecionado concordou e foi submetido a CRVP.

TC6M foi realizada em 19 pacientes resultando em distância média percorrida de 355 metros (mínima de 175 metros e máxima de 535 metros).

Vinte e oito pacientes realizaram TCAR resultando em um grau de lesão pulmonar médio de 48,9l com o menor valor de 20 e o maior de 79.

Todos os pacientes realizaram espirometria e gasometria arterial (ar ambiente), resultando em CVF média de $74 \%$ do previsto $(32 \%$ - $130 \%)$; VEFI médio de $41 \%$ (16\% - $124 \%$ ) e relação VEFI/CVF média de $44 \%(24 \%-77 \%)$. $\mathrm{PaO} 2$ média $=70,18$ $\mathrm{mmHg}$; $\mathrm{PaCO} 2$ média $=43,6 \mathrm{mmHg}$ e $\mathrm{pH}$ médio $=7,33$.

\section{Discussão}

O principal critério para seleção de pacientes a serem submetidos à CRVP é a presença de doença com comprometimento parenquimatoso heterogêneo, predominando nos lobos superiores bilaterais ${ }^{6}$.

Dentre as principais indicações para a realização da CRVP podemos citar: dispnéia incapacitante com piora na qualidade de vida, VEFI entre $25 \%$ e $35 \%$ do previsto, hiperinsuflação pulmonar com capacidade pulmonar total maior que $120 \%$ e relação volume residual/capacidade pulmonar total maior que $60 \%{ }^{6}$.

Quanto aos critérios de contra-indicação à realização da CRVP, podem-se citar: pacientes hipersecretivos caracterizando bronquite crônica, idade superior a 80 anos, cardiopatia ou outra comorbidade grave, hipertensão arterial pulmonar maior que $35 \mathrm{mmHg}$, hipercapnia grave ( $\mathrm{PaCO} 2$ acima de $55 \mathrm{mmHg}$ ), história de doença neoplásica prévia, cirurgia torácica prévia, dependência de ventilação mecânica, VEFI maior que $35 \%$ ou menor que $25 \%$ do previsto, volume residual menor que $150 \%$ do predito, ansiedade edepressão incontroláveis, peso corporal acima de $140 \%$ do peso ideal, deficiência da enzima alfa-I- antitripsina e pacientes tabagistas 6 .

Dos pacientes contra-indicados, nove (29\%) apresentavam brônquite crônica. Existe consenso em contra-indicar a realização da CRVP em pacientes hipersecretivos. Sabe-se que estes não evoluem satisfatoriamente no pós operatório, tendem a apresentar com maior freqüência infecções respiratórias que aumentam a morbidez e a mortalidade.

Quatro pacientes não foram operados pois apresentavam na avaliação pré operatória VEFI acima dos valores recomendados, nãose justificando os riscos do ato cirúrgico.

Três pacientes, apesar de selecionados, recusaram-se a se submeter à CRVP. $O$ primeiro, um paciente masculino de 64 anos, VEFI de $26 \%$ do previsto e percorrendo ao TC6M 365 metros e TCAR revelando enfisema pulmonar com predomínio apical bilateral sem contra indicações paraa CRVP. Ao ser submetido ao tratamento clínico e ao programa de reabilitação pulmonar referiu melhora, recusando-se submeter à cirurgia. $O$ segundo paciente, masculino de 76 anos, diagnosticado enfisema pulmonar heterogêneo com predomínio nos ápices, VEFI de $670 \mathrm{~mL}$ ( $28 \%$ do previsto) e desempenho no TC6M de 290 metros. Apesar destas características favoráveis, e da ausência de qualquer outra contra indicação, não aceitou a proposta de ser submetido à CRVP após referir alguma melhora com o programa de reabilitação pulmonar. $O$ terceiro paciente, apesar de características favoráveis, recusou-se a realizá-la antes mesmo que pudesse ser submetido a um programa de reabilitação.

Dois pacientes foram contra-indicados à CRVP pois detectou-se a presença de fibrose pulmonar cicatricial na tomografia computadorizada de tórax. O primeiro apresentava história patológica pregressa de tuberculose pulmonar, com áreas fibrosadas em ápices visualizadas no estudo imagético. Mostrou também um desempenho ruim no TC6M, 
percorrendo apenas 200 metros. 0 segundo, um paciente masculino de 58 anos de idade, também apresentava história patológica pregressa de tuberculose pulmonar, VEF I de $0,67 \mathrm{~L}$ (19\% do previsto) e 190 metros percorridos durante o TC6M. Áreas de fibrose pulmonar são responsáveis por aderências pleurais as quais criam dificuldades para a realização de procedimentos cirúrgicos intratorácicos.

Dois pacientes apresentaram quadros depressivos. $O$ primeiro, um homem de 53 anos com VEFI de $700 \mathrm{~mL}$ ( $26 \%$ do volume previsto) e hipoxêmico ( $\mathrm{PaO} 2=51,5 \mathrm{mmHg})$. Apresentava-se bastante desmotivado e deprimido, manifestando idéias suicidas enegando-se a fazer acompanhamento psiquiátrico, sendo descartado como candidato à CRVP. A segunda paciente, uma mulher de $7 \mathrm{I}$ anos de idade e portadora de enfisema pulmonar, com predomínio apical a TCAR e VEFI 33\% do volume previsto, foi encaminhada ao psiquiatra apresentando melhora do quadro depressivo. Sendo novamente indagada quanto à realização da cirurgia demonstrou interesse, porém solicitou um prazo para que pudesse consultar os filhos, os quais não permitiram que fosse submetida à cirurgia.

Dois pacientes apresentavam bron-quiectasias. Estes pacientes apresentam quadros hipersecretivos e conseqüentemente um comportamento semelhante ao daqueles com bronquite crônica.

A presença de nódulo neoplásico pulmonar por si só não representa uma contraindicaçãoà CRVP, desde que se trate de doença localizada e posicionada em topografia compatível comáreas de destruição enfisematosa a serem ressecadas para se completar a redução. Na presente amostra, uma paciente apresentava um nódulo pulmonar cuja investigação demonstrou tratar-se de um carcinoma. Infelizmente, foi detectada a presença de metástase óssea, o que inviabilizou a sua ressecção com CRVP simultânea.

Um paciente apresentou na TCAR extenso enfisema bolhoso, representando um sério problema técnico ealto risco do tecido pulmonar remanescente não ser suficiente para ocupar o espaço antes ocupado pelo tecido doente ressecado, o que acarreta fugas aéreas prolongadas.

Um paciente apresentava quadro de obesidade mórbida (índice de massa corporal igual a44). Tratava-se de paciente dispnéico, hipoxêmico $(\mathrm{PaO} 2=56,9 \mathrm{mmHg})$, hipercápnico $(\mathrm{PaCO} 2=52,0 \mathrm{mmHg})$ e com distúrbio obstrutivo grave (VEFI = $720 \mathrm{~mL}$ com $25 \%$ do valor previsto), porém os transtornos produzidos pela elevada massa corporal representaram contra-indicação a CRVP.

Um dos pacientes apresentou sinais clínicos de hipertensão arterial pulmonar com gasometria arterial demonstrando retenção de $\mathrm{CO}$. Este paciente acusou durante os exames de rotina a presença de ovos de Schistossoma mansoni em suas fezes. Retenção de $\mathrm{CO} 2$ acima de $55 \mathrm{mmHg}$ e quadros de hipertensão arterial pulmonar (HAP) são contra-indicações à realização de CRVP. Evidências laboratoriais de HAP (ecocardiograma transtorácico bidimensional com Doppler e trans-esofágico), associado a hipercapnia e um possível envolvimento pulmonar pela schis-tossomose representaram as principais contra-indicações da CRVP.

Um dos pacientes analisados pode ser descartado para a realização de CRVP devido à hístória patológica pregressa de haver sido submetido à toracotomia para ressecção de nódulo pulmonar neoplásico há quatro anos. $\mathrm{A}$ realização de toracotomia prévia representa contra-indicação pelas dificuldades técnicas representadas por aderências pleurais que impedem a manutenção da integridade pleural necessária para o sucesso do tratamento. Esta cirurgia havia sido realizada devido à presença de uma neoplasia pulmonar há quatro anos, representando outra contra-indicação, descartando-se sua realização contralateral. Posteriormente ao longo da avaliação foi encontrada, outra contra indicação: apresentava VEFI de $43 \%$ do volume previsto.

Um paciente apresentou durante a avaliação enfisema pulmonar difusamente distribuído (enfisema homogêneo) como contra indicação. Tratava-se de um homem de 46 anos, VEFI de $580 \mathrm{~mL}$ ( $25 \%$ do volume previsto), limitação de atividade física verificada no TC6M, no qual se constatou uma distância percorrida de 335 metros. Constatou-se, durante a avaliação, que este paciente apresentava deficiência de alfa-I-antitripsina. Infelizmente, apresentou na cintilografia perfusional um comprometimento pulmonar bilateral e difuso.

Um paciente masculino de 56 anos com extenso enfisema pulmonar bolhoso e impor- tante queixa de dispnéia teve a contra-indicação formalizada, pois era tabagista e, ao ser indagado, negou o desejo de abandoná-lo. Como o tabagismo é o principal fator etiológico no desenvolvimento do enfisema pulmonar, há concordância entre todos os autores da necessidade de sua abstinência por pelo menos seis meses antes de se proceder a CRVP. Apesar da ampla destruição do parênquima pulmonar (grau de lesão igual a 63), apresentava uma espirometria com VEFI = I.34 litros (60\% do valor previsto), o que representava outro fator a descaracterizá-lo como candidato à CRVP.

Um paciente masculino, de 45 anos de idade, com VEFI de $690 \mathrm{~mL}$ (33\% do previsto) e enfisema heterogêneo a TCAR com predomínio do terço médio e superior bilateralmente, apresentou durante o TC6M, dor precordial, obrigando a interrupção do exame devido à suspeita de isquemia cardíaca.

O paciente que concordou, e realizou a CRVP, apresentava doença com comprometimento parenquimatoso heterogêneo, predominando nos lobos superiores e bilaterais. Segundo McKenna et al. ${ }^{6}$ este é o principal critério para um paciente com enfisema ser submetido ao tratamento cirúrgico realizado. Evoluiu sem morbidades e sem mortalidade, no pós-operatório imediato, e teve melhora clínica, laboratorial e qualidade de vida, durante três anos de acompanhamento ambulatorial?

\section{ConClusões}

São múltiplos e complexos os fatores que contra-indicam a CRVP. Bronquite crônica representou a principal contra-indicação em $29 \%$ dos pacientes. É comum que pacientes encaminhados para a realização da CRVP apresentem mais de umfator mórbido a contra-indicar oseu procedimento. Entre os pacientes que se encaixam nos critérios de realização de CRVP existe grande receio de se submeter à mesma com a maioria se recusando a realizá-la após a realização de programas de reabilitação pulmonar.

\section{Agradecimentos}

Ao professor Luiz PauloSantos Verbicário pela criação do Ambulatório Multidisciplinar de DPOCe Tabagismo da Universidade Federal Fluminense. Seus esforços, dedicação e competência, colaboraram, de modo significativo, paraa realização deste ede outros trabalhos de pesquisa. 


\section{SUMMARY}

LUNG VOLUME REDUCTION SURGERY (LVRS): CRITERIA FOR SELECTING PATIENT in Antonio Pedro Hospital, Federal Fluminense U niversity, Niteról city, Rio de Janeiro, Brazil

BACKGROUND. Chronic Obstructive Pulmonary Disease (CPOD) is an important problem in Brazil and other countries. The clinical treatment of ambulatory patients is now reasonably standardized. Lung volume reduction surgery (LVRS) is an alternative of surgical treatment of emphysema (aside from lung transplantation).

OBJECTIVES. The aim of this study was to analyse patient selection criteria for LVRS.

METHODS. This study was carried out using a sample of 31 patients referred to and given assistance to at the Smoking and Chronic Obstructive Pulmonary Disease Outpatient Ward Antonio Pedro Hospital, who were evaluated to check whether or not they could be eligible for LVRS. Aiming at evaluating the major criteria for eligibility for LVRS, the patients underwent a protocol including standardized anamnesis and physical examination, spirometry with maximum inspiratory and expiratory flow volume curves associated with post-bronchodilatator test, room air arterial blood gases, a six-minute walking test, plain chest roentgenograms and left lateral chest roentgenograms, high-resolution chest com- puted tomographic scans and lung perfusion scans. Statistical analysis was conducted with description of data proportions.

RESULTS. Contraindications to surgery included chronic bronchitis ( $29 \%$ or nine patients), forced expiratory volume in one second (FEV I) more than 80 percent predicted ( $13 \%$ or four patients), negative consent for surgery $(10 \%$ or three patients), fibrosis pattern, severe depression and bronchiectasis (two patients each one), solitary pulmonary nodule, severe obesity, severe bullous emphysema, pulmonary hipertension, pulmonary resection history, current cigarette smoking, diffuse emphysema with alpha-I-antitrypsin deficiency and severe cardiac disease (one patient each). Indication. One selected patient with severe symptoms of emphysema met criteria for LVRS (bilateral superior lobe heterogeneous pattern of emphysema on chest computed tomography and lung perfusion scan).

CONCLUSIONs. Chronic bronchitis was the major isolated factor to contraindicate LVRS. Most patients who fit the criteria for the surgery performance refused to undergo the LVRS for fear of the surgery and after pulmonary rehabilitation. The most important criteria for LVRS is bilateral superior lobe heterogeneous pattern of emphysema. [Rev Assoc Med Bras 2003; 49(3): 274-7]

KEY WORDS. Lung volume reduction surgery. Emphysema.

\section{REFERÊNCIAS}

I. Brantigan OC, Mueller E, Kress MB. A surgical approach to pulmonary emphysema. Am Rev Respir Dis 1959; 80:194-202.

2. Laforet EG. Surgical management of chronic obstructive lung disease. N Engl J Med I972; 287: 175-7.

3. Cooper JD, Trulock EP. Bilateral pneumonectomy. (volume reduction) for chronic obstructive pulmonary disease. J Thorac Cardiovasc Surg; 1995; 109: 106- 16.

4. Lima OAS, Verbicário LPS. Redução Unilateral de Volume Pulmonar. In: Sociedade de Pneumologia e Tisiologia do Rio de Janeiro. Pneumologia: aspectos práticos e atuais. Rio de Janeiro: Revinter; 2001.

5. Bergin C, Muller N, Nichos DM, Lillington G, Hogg JC, Mullen B, et al. The diagnosis of emphysema. Am Rev Respir Dis 1986; |33:541-6.

6. Mckenna RJ, Brenner M, Fischel RJ, Singh N, Yoong B, Gelb AF, et al. Patient selection criteria for lung volume reduction surgery. J Thorac Cardiovasc Surg 1 997; I | 4:957-64.

7. Behrsin RF. Cirurgia redutora de volume pulmonar: critérios de seleção de pacientes no Hospital Universitário Antônio Pedro (dissertação). Niterói: Universidade Federal Fluminense; 1999.

Artigo recebido: 09/08/02 Aceito para publicação: 09/10/02 